$\frac{12}{12} / 292880$

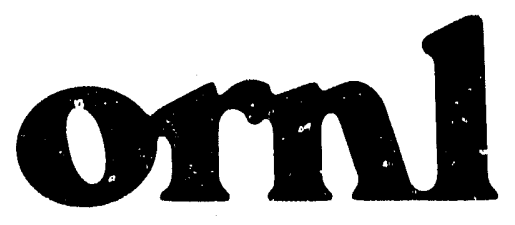

ORNL/TM-12005

OAK RIDGE NATIONAL LABORATORY

\section{Atom Probe Field Ion Microscopy and Related Topics: A Bibliography 1990}

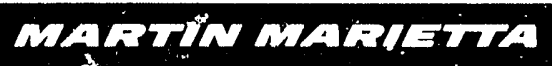

K. F. Russell

M. K. Miller 
This report has been reproduced directly from the best available copy.

Available to DOE and DOE contractors from the Office of Scientific and Technical Information, P.O. Box 62, Oak Ridge, TN 37831; prices avallable from (615) 576-8401. FTS 626-8401.

Available to the public from the National Technical Information Service, U.S. Department of Commerce, 5285 Port Royal Rd., Springfield, VA 22161.

NTIS price codes-Printed Copy: A07 Microfiche A01

This report was prepared as an account of work sponsored by an agency of the United States Government. Neither the United States Government nor any agency therecif, nor any of their employees, makes any warranty, express or implied, or assumes any legal liability or responsibility for the accuracy, completeness, or usefulness of any information, apparatus, product, or process disclosed, or represents that its use would not infringe privately owned rights. Reference herein to any specific commercial product, process, or service by trade name, trademark, manufacturer, or otherwise, does not necessarily constitute or imply its endorsement, recommendation, or favoring by the United States Government or any agency thereot. The views and opinions of authors expressed herein do not necessarily state or reflect those of the United States Government or any agency thereof. 
ORNL/TM- -12005

DE92 005518

Metals and Ceramics Division

ATOM PROBE FIELD ION MICROSCOPY AND RELATED TOPICS:

A BIBLIOGRAPHY 1990

K. F. Russell

M. K. Miller

Date Published - December 1991

Prepared for the

DOE Office of Basic Energy Sciences

KC 0201010

Prepared by the

OAK RIDGE NATIONAL J.ABORATORY

Oak Ridge, Tennesses 37831-6285

managed l,y

MARTIN MARIETTA ENERGY SYSTEMS, INC.

for the

U.S. DEPARTMENT OF ENERGY

under contract DE-AC05-84OR21400 


\section{PREFACE}

This bibliography includes references related to the following topics: atom probe field ion microscopy (APFIM), field ion microscopy (FIM), field emission (FF), ion sources, and field desorption mass microscopy (FDMM). Technique-orientated studies and applications are included.

This bibliography covers the period 1990. Previous publications, Atom Probe Field-ion Microscopy and Related Topics: A Bibliography 1978-1987, compiled by M. K. Miller and A. R. McDonald, ORNL/TM-11157; Atom Probe Field-Ion Microscopy and Related Topics: A Bibliography 1988, compiled by M. K. Miller and A. R. Hawkins, ORNL/TM11370; Atom Probe Field Ion Microscopy and Related Topics: A Bibliography 1989, compiled by M. K. Miller, A. R. Hawkins, and K. F. Russell, ORNL/TM-11696, Oak Ridge National Laboratory, Oak Ridge, TN 37831-6285; and Field-Ion Microscopy and Related Techniques, A Bibliography: 1951-1978, compiled by R. E. Thurstans and J. M. Walls, published by Warwick, Birmingham, contain the papers published prior to this period.

The references contained in this document were compiled from a variety of sources including computer searches and personal lists of publications. To reduce the length of this document, the references have been reduced to the minimum necessary to locate the articles. The references, listed alphabetically by authors, are subdivided into the categories listed in paragraph one above. An Addendum of references missed in previous biblicgraphies is included.

We would like to thank Dr. G. D. W. Smith of Oxford University, Dr. L. L. Horton of Oak Ridge National Laboratory (ORNL), and M. Alexander of the ORNL Central Library for their stoic efforts in the preparation of this document. We would also like to thank the many authors for supplying their personal publications lists, and we apologize for any errors or omissions that may have occurred in compiling this document.

K. F. Russell and M. K. Miller Metals and Ceramics Division 


\section{CONTENTS}

1990

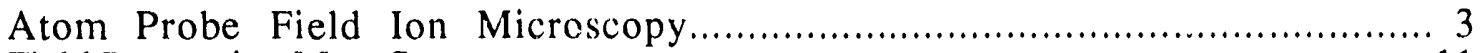

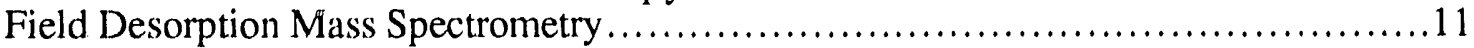

Field Emission ........................................................................ 13

Field Ion Microscopy........................................................ 19

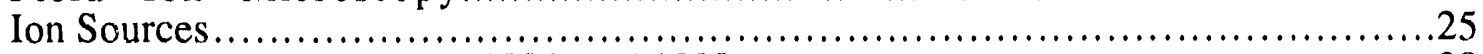

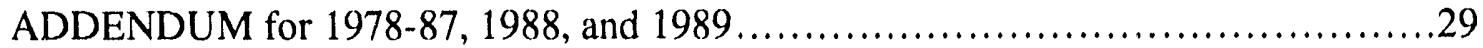

APPENDIX 
1990 
1 The effect of aging at $343^{\circ} \mathrm{C}$ on type 308 stainless steel weldments

2 Microscopical evaluation of low temperature aging of type 308 stainless steel weldments

3 Atom probe and transmission electron microscopy study of aging of cast duplex stainless steels

4

Fine scale investigation of some phenomena in metallic alloys by field ion microscopy and atom probe microanalysis

$5 \quad$ On grain boundary phases in B-doped $\mathrm{Ni}_{3} \mathrm{Al}$

6 Grain boundary segregation of carbon and boron in $\mathrm{Ni}_{3} \mathrm{Al}+\mathrm{B} / \mathrm{C}$

7 Quantitative atom probe analysis of spinodal reaction in ferrite phase of duplex stainless steel

The thermal ageing of alloys modelling the ferrite phase in stainless stecl castings
Alexander, D. J. Alexander, K. B. Miller, M. K. Nanstad, R. K.

Alexander, K. B. Miller, M. K.

Alexander, D. J. Nanstad, R. K.

Auger, $\mathrm{P}$.

Danoix, $F$.

Menand, A.

Bonnet, S.

Bourgoin, J.

Guttman, M.

Blavette, D.

Auger, $P$.

Brenner, S. S.

Hua, M.-J.

Brenner, S. S,

Hua, M.-J.

Brown, J. E.

Cerczo, A.

Godfrey, T. J.

Hetherington, M. G.

Smith, G. D. W.

Brown, J. E.

Hetherington, M. G.

Smith, G. D. W.

Pumphrey, P. H.
Fatigue, Degradation and Fracture, ASME Symp. Proc. (1990) PVP 195, MPC 30 , 187-92, Banford, W. H., Becht, C., Bhandari, S., Gilman, J. D., James, L. A., and Prager, M., eds., ASME, New York

Mater. Sci. Technol. (1990) ‥ 314-20

Mater. Sci. Technol. (1990) 6. 301-13

Microsc. Microanal. Microstruct. (1990) 1 . 481-92

Scr. Mctall. (1990) 24 . 671-6

Scr. Mctall. (1990) 24. 667-70)

Mater. Sci. Technol. (1990) 6. 293-300

Fatigue, Degradation and Fracture, ASME Symp. Proc, (1990) PVP 195, MPC 30. 175-85, Bamford, W. H., Becht, C., Bhandari, S., Gilman, J. D., James, L. A., and Prager, M., eds., ASME, New York 
9 Advances in microscopy of materials Brown, L. M.

10 An analysis of small clusters formed in thermally aged and irradiated $\mathrm{FeCu}$ and $\mathrm{FeCuNi}$ model alloys

11 Atom probe field-ion microscopy applications

12 AP/FIM study of multicomponent $\mathrm{M}_{2} \mathrm{C}$ precipitation

13 The position sensitive atom probe: three dimensional reconstruction of atomic chemistry

Cerezo, A.
Bu;well, J. T. Entrlish, C. A. Hetherington, M. G. Phythian, W. J. Smith, G. D. W. Worrall, G. M.

Camus, P. P.

Carinci, S. M.

Olson, G. B. Liddle, J. A. Chang, L. Smith, G. D. W.
Mater. Sci. Technol. (1990) 6, 967.73

Effects of Radiation on Materials: 14th Intemational Symposium, ASTM STP 1046, II, (1990) 127-53, Packan, N. H., Stoller, R. E., and Kumar, A. S., eds., ASME, Philadelphia, PA

High Temp. Sci. (1990) Vol. Datc 1988-89, 26. $131-42$

Innovations in Ultrahigh-Strength Stecl Technology, Sagamore. Army Materials Rescarch Conf. 134th: 1987: Lake George, NY] (1990) 179-208, Olson, G. B., Azrin, M., Wright, E. S., eds., US Army Materials Research Laboratory, Watertown, MA

Godfrey, T. J.

Grovenor, C. R. M. Hetherington, M. G. Hyde, J. M. Liddle, J. A. Mackenzic, R. A. D. Smith, G. D. W.

Cerezo, A.

Grovenor, C. R. M. Hetherington, M. G. Sha, W.

Shollock, B. A.

Smith, G. D. W.
Electron Microsc. Soc. Am. Bull. (1990) 20:2, 77-83
Mater. Charact. (1990) 25. 143-56
14 Analysis of nanometer-sized precipitates using atom probe techniques 
15 Atom probe and HREM characterization of transition metal multilayers

16 Embrittiement of laboratory and reactor aged $\mathrm{CF} 3, \mathrm{CF} 8$, and $\mathrm{CF} 8 \mathrm{M}$ duplex stainless steels

17 Solute-atom segregation at internal interfaces

18 Investigations on phase transformations and $\mathrm{B} 2-\mathrm{DO}_{3}$ superlattices in ordered iron-aluminum alloys by FIM-atom probe and TEM

19 An image processing facility specialized for the FEM-FM-IAP system

20) Atomic-scale observations of two-dimensional Re segregation at an internal interface in $\mathrm{W}(\mathrm{Re})$

21 Atom-probe micro-characterization of local composition fluctuations and passivated surface of stainless siecl

22 Analysis of small precipitates in high-strength, low-alloy stecls by atom-probe field-ion microscope

23 A system for systematically preparing atom-probe field-ion microscope specimens for the study of internal interfaces
Cerezo, A.

Hetherington, M. G. Petford-Long, A. K.

Chung, H. M.

Leax, T. R.

Foiles, S. M.

Seidman, D. N.

Frommeyer, G.

Habrock, H.-J.

Wittig, J. E.

Von Czamowski, P.

Geenen, J.

Kreuss, $M$.

Hu, B. Y.

Liu, W.

Ren, D. M.

Xiao, S. S.

Hu, J. G.

Scidman, D. N.

Ishikawa, Y.

Koguchi, Y.

Yoshimura, $\mathrm{T}$.

Kucissl, A.

Brenner, S. S.

Krakaucr, B. W.

Hu, J. G.

Kuo, S.-M.

Mallick, R. L.

Scki, A.

Seidman, D. N.

Baker, J. P.

Loyd, R. J.
Thin Film Structures and Phase Stability, Mat. Res. Soc. Symp. Proc. (1990) 187, 333-8, Clemens, B. M. and Johnson, W. L., eds., MRS, Pittsburgh, PA

Mater. Sci. Technol. (1990) 6. 249-62

Mater. Res. Soc. Bull. (1990) 15(9), 51-7

Scr. Metall. (1990) 24 , 51-6

J. Electron. Sci. Tech. (Chinese) Suppl. I (1990) 253

Phys. Rev. Lett. (1990) 65 . 1615-8

Boshoku Gijutsu (1990) 39(5), 232-7

Prakı. Mctallogr. (1990) 27(4.), 159-70)

Rev. Sci. Instrum. (1990) $3.390-8$ 
24 Atom probe with space-time ion focusing

25 Solute-atom segregation-an oscillatory Ni profile at an internal interface in $\mathrm{Pt}(\mathrm{Ni})$

26 Combined study of multiple-quantum-well structures using high resolution electron microscopy imaging and analysis in conjunction with the position sensitive atom probe

27 A miniature electron-beam evaporator for an ultrahigh-vacuum atom-probe field-ion microscope

28 Several key assemblies specially designed for the FEM-FIM-IAP system

29 The advantages of APFIM in the study of suriace chemical processes

30 The study of elementary steps of surface chemical process by using pulsed laser atom probe

31 The first pulsed laser atom probe in China

32 The surface structure effect on the chemisorption of $\mathrm{Co}$ on $\mathrm{Rh}$
Kudryavtsev, A. N. Nikonenkov, N. V. Dubenskii, B. M.

Shmikk, D. V.

Kuo, S.-M.

Scki, A.

Oh, Y.

Seidman, D. N.

Liddle, J. A.

Long, N. J.

Petford-Long, A. K.

Lin, X. W.

Hu, J. G.

Seidman, D. N.

Morikawa, $\mathrm{H}$.

Liu, W.

Chen, H. N.

Liu, L. M.

Ren, D. M.

Liu, W.

Ren, D. M.

Liu, W.

Ren, D. M.

Liu, W.

Ren, D. M.

Huang, G. M.

Chen, H. N.

Liu, L. M.

Liu, W/u.

Ren, D. M.
Instrum. Exp. Tech. (1990) 33(2), 388-91

Phys. Rev. Lett. (1990) 65 , 199-202

Mater. Charact. (1990) 25 , 157-76

Rev. Sci. Instrum. (1990) 61. 3745-9

J. Electron. Sci. Tech. (Chinese) Suppl. 1 (1990) 250

International Seminar on STM and FIM, March 26-28, 1990, Beijing Lab. of Vacuum Phys., Beijing, China

J. Electron. : ici. Tech. (Chinese) Suppl. 1 (1990) 171

J. Electron. Sci. Tech. (Chinese) Suppl. 1 (1990) 22

Surf. Sci. (1990) 232, 323-8 
33 The atomic structure of A1-B2 interfaces in a $\mathrm{Ni}-\mathrm{Be}$ Alloy

34 The structure of the $\gamma^{\prime}$-precipitates and the precipitation hardening of an Elinvar alloy

35 Pulsed laser atom probe analysis of III-V compound semiconductor epilayers
Liu, Z. G.

Al-Kassab, T.

Haasen, $\mathrm{P}$.

Liu, Z. G.

Al-Kassab, T.

Haasen, $P$.

Mackenzie, R. A. D.

Liddle, J. A.

Grovenor, C. R. M.

Cerezo, A.
Acta Mclill. (1990) 38 . 1857-62

Scr. Metall. (1990) 24 , 65.5-60

Layered Structures Hetcrocpitaxy, Supcrlattices, Strain and Mctastability, Mat. Res. Soc. Symp. Proc. (199')) 160, 439-44, Dodson, B. W., Schowalter, L. W., Cunningham, J. E., and Pollak, F. H., eds., MRS, Pittsburgh, PA

Mackenzic, R. A. D. Smith, G. D. W.

Cerezo, A.

Godfrey, T. J.

Brown, J. E.

Mackenzic, R. A. D. Smith, G. D. W.

Cerezo, A.

Liddle, J. A.

Grovenor, C. R. M.

Hetherington, M. G.

Miller, M. K.

Bentlcy, J.

Miller, M. K.

Burke, M. G.
Proc. 12th Intl. Congress on Electron Microscopy, Seattle (1990), 4, 408-9, San Francisco Press, San Francisco, CA

Proc. 12th Intl. Congress on Elcctron Microscopy, Scattle (1990), 4, 624-5, San Francisco Press, San Francisco, CA

Mater. Sci. Technol. (1990) 6, 285-92

Effects of Radiation on Matcrials: 14th International Symposium, ASTM STP 1046, II, (1990) 107-26, Packan, N. H., Stoller, R. E., and Kumar, A. S., eds., ASTM, Philadelphia, PA

Scr. Metall. (1990) 24 . ultrafine isotropic microstructures in iron-chromium alloys from atom probe ficld ion microscopy data
Miller, M. K.

Hetherington, M. G.

\author{
1375-80
}


41 The oxidation of nickel zirconium ( $\mathrm{Ni}_{3} \mathrm{Zr}$ ) investigated by atom-probe field-ion microscopy and $X$-ray photoelectron spectroscopy

42 On influence of cobalt on structure and phase transformations during tempering of carbon-containing stecls of Fe-Ni-Mo system

43 Study of the process of field evaporation in nickel-aluminum $\left(\mathrm{Ni}_{3} \mathrm{Al}\right)$ alloys as Markov chains

44 Study of the process of field evaporation in nickcl-aluminide $\left(\mathrm{Ni}_{3} \mathrm{Al}\right)$ alloy as Markov chains

45 A study of chemisorption behavior of carbon monoxide on rhodium surfaces

46 The vacuum system of the FEM-FIM-IAP system

47 Surface segregation of Pt-Rh and $\mathrm{Pt}-\mathrm{Ru}$ alloys

48 Surface segregation of platinum-rhodium alloys

49 Atomic resolution studies of solute-atom segregation at grain boundaries: experiments and Monte Carlo simulations
Perry, E. M. Cocke, D. L. Miller, M. K.

Rakhshtadt, A. G. Khovova, O. M.

Kan, A. V.

Perkas, M. D.

Kudryavtsev, A. N.

Rodionov, Yu. L.

Ren, D. G.

Wang, Q.

Guo, J. T.

Ren, D. G. Wang, Q.

Guo, J. T.

Ren, D.

Liu, W.

Ren, D. M.

Liu, L. M.

Huang, G. M.

Chen, H. N.

Liu, W.

Ren, D. M. Tsong, $T$. T. Liu, W.

Qin, J. $\mathrm{H}$.

Sano, N.

Sakurai, T.

Scidman, D. N.

Hu, J. G.

Kuo, S.-M.

Krakauer, B. W.

Oh, Y.

Scki, A.
Appl. Surf. Sci. (1990) 44 .

$321-30$

Fiz. Met. Metalloved. (195,0)

(7), 77-87

Rarc Met. (Bcijing), (1990) 2(3), 189-93

Scr. Metall. (1990) 24 , 1707-12

Surf. Sci. (1990) 232 .

316-22

J. Electron. Sci. Tech. (Chincse) Suppl. 1 (1990) 258

J. Electron. Sci. Tech. (Chinese) Suppl. 1 (1990) 166

J. Vac. Sci. Technol. (1990) A8, $3421-4$

J. de Physique (1990) 51.

C1-47-57 
50 The position sensitive atom probe: a review

51 Atom probe studies of some maraging stecls

52 Compositional studies of $\Omega$ and $\theta^{\prime}$ precipitates in an $\mathrm{Al}-\mathrm{Cu}-\mathrm{Mg}-\mathrm{Ag}$ alloy

53 The distribution of substitutional alloying elements during the bainite transformation

54 Quantitative surface analysis atomic resolution, atom-probe ficld ion microscopy

55 Energetics of surface atomic processes

56 Oscillatory surface cosegregation of $\mathrm{Pt}-\mathrm{Rh}(\mathrm{S})$ alloys

57 Impurity adsorption induced surface charge-density oscillation and indirect atomic interactions

58 Solid state precipitation at high supersaturations
Sha, W.

Cerezo, A.

Godfrey, T. J.

Smith, G. D. W.

Sha, W.

Cerezo, A.

Smith, G. D. W.

Shollock, B. A.

Grovenor, C. R. M.

Knowles, K. M.

Stark, I.

Smith, G. D. W.

Bhadeshia, H. K. D. H.

Tsong, T. T.

Tsong, T. T.

Tsong, $T$. $T$.

Ahmad, $\mathrm{M}$.

Tsong, $T$. T.

Chen, C. L.

Wagner, R.

Kampmann, R.
J. Electron. Sci. Tech. (Chinese) (1990), Suppl. 1, 17-21

J. Electron. Sci. Tech. (Chinese) (1990), Suppl. 1, 142-7

Scr. Metall. (1990) 24, 1239-44

Mctall. Trans. (1990) A21, 837-44

J. Vac. Sci. Technol. (1990) A8, 3397-404

Surf. Sci. (1990) 231, $81-9$

Phys. Rev. B (1990) 42 . 1464-6

Mod. Phys. Lett. B (1990) 4. 775-82

Innovations in Ultrahigh-Strength Steel Technology, Sagamorc, Army Materials Research Conf. [34th: 1987: Lake Gcorge, NY] (1990)

Olson, G. B., Azrin, M. Wright, E. S., eds., US Army Materials Research Laboratory, Watertown, MA

Wang, Q.

Kinkus, T. J. Ren, D. G.
Chin. Phys. Lett. (1990) 7(8), 353-6 
60 Atom-probe field-ion microscope study of amorphous structure: Markov chain analysis

61 Atom probe microcharacterization of native oxide on silicon

62 Atom-probe characterization of aged stainless steels
Yamamoto, M. Kawabata, K.

Nenno, S.

Ohnaka, I.

Yoshimura, T. Ishikawa, Y.

Yoshimura, T. Ishikawa, Y.
J. Non-Cryst. Solids (1990) $117,152-5$

Solid-State Electron.

(Suppl.) (1990) 33, 321-6

Microstruct. Evol. Met. Process., PED-ASME

(1990) 46 . 77-87 
1 Smearing method for preparation of MSFD/FI emitters and their performance

2 On the formation of doubly charged fragment and cluster ions of oxygenand sulfur-containing substances in field ionization and field desorption mass spectrometry

3 Time-resolved reaction studies on the atomic scale: nitric oxide and carbon monoxide adsorption on slepped rhodium surfaces

4 Methanol decomposition on Pd (111) single crystal surfaces
$\mathrm{Hu}, \mathrm{T}$.

Zhao, M.

Wei, $\mathrm{H}$.

Kosevich, M. V.

Shelkovskii, V.S.

Krusc, N.

Krusc, N.

Rebhol\%, M.

Matolin, V.

Chuah, G. K.

Block. J. H.
Fenxi Ceshi Tongbao (1990) 9. $11-15$

Rapid Commun. Mass Spectrom. (1990) 4 (12). 493-4

J. Vac. Sci. Technol. (1990) A8, 3432-6

Surf. Sci. Lell. (1990) 238. L457-62 
Current fluctuation of field emission cathodes

2 Coverage dependence of field-emission flicker noise due to lithium adsorbed on the W(112) surface

3 Use of ficld-emission microscopy for investigation of the corrosion of metals and metal-film systems

Electron source with micropoint emissive cathodes and display means by cathodoluminescence excited by ficld cmission using said source

5 Ficld emission studies on technical niobium surlaces

6 Scanning electron and ficld emission microscopy of supported metal clusters

7 A field emission technique to measure the melting temperature of individual nanometer-sized clusters

8 Ficld emitter tips for vacuum microclectronic devices

9 Diffusion of tungsten on a tungsten (211) planc

10 Magnitude and its temperature dependence of the cross-correlation factor of flicker noise from the potassium/tungsten (110)
Adachi, $\mathrm{H}$.

Biernat, $\mathrm{T}$. Klcint, C.

Bobkov, A. F.

Gogoberidzc, M. I.

Klimiashvili, L. D.

Suvorov, A. L.

Borcl, $\mathrm{M}$.

Boronat, J. F.

Meycr, R.

Rambaud, P.

Calder, R. S.

Dominichini, G.

Hilleret, N.

Castro, T.

Li, Y. Z.

Reifenberger, $\mathrm{R}$.

Choi, E.

Park, S. B.

Andres, R. P.

Castro, $T$.

Reifenberger, $\mathrm{R}$.

Choi, E.

Andres, R. P.

Chin, K. K.

Marcus, R. B.

Choi, D.-S.

Gomer, R.

Dabrowski, A. M.
Shokubai (1990) 32(7). 481-7

Appl. Phys. A. (1990) 50, 95-100

Prot. Mct. R. (1990) 26(4), 477-80

U.S. Patent No. 4940916

(1990)

IEEE Trans. Electr. Insul. (1990) 25, 363-6

ACS Symp. Scr. (1990) 437 (Novel Mater. Helcrog. Catal.), 329-41

Surf. Sci. (1990) 234, 43-52 A $8,3586-90$

Surf. Sci. (1990) 230. $277-82$

Acta Phys. Pol. (1990) A78, 775-80 
11 Observation of electron-hole cascade in photoficld emission

12 Codeposition of aluminum oxide and silicon oxide on a single crystal tungsten surface

13 Destabilization mechanisms in lield-emission structures with small vacuum gap

14 Coherent point source electron beams Fink, H.-W. Stocker, W. Schmid, $\mathrm{H}$.

15 What do we mean by "work function"?
Donders, P. J.

Lec, M. J. G.

Dracger, N. A.

Eremohen., D. V

Makhov, V. I.

Forbes, R. G.

Fursei, G. N

Kocheı yzhenkov, A. V.

Maslov, V. I.

Shmaev, A. L.

Borisov, L. N.

Ganguly, A. K. Phillips, P. M.

Gray, H. F.

Ghatak, K. P. Biswas, S. N. telluride-cadmium telluride superlattices with graded structures under magnetic quantization

Linear theory of a fied
distributed amplifier

18 On the field emission from mercury

19 Ficld emission from superlattices of small-gap materials with graded structures in the presence of a strong magnetic ficld

20 Kinetic approach to field emission from semiconductors by computer simulation using particles
Phys. Rev. B (1990) 41 1781-8

J. Cryst. Growth (1990) 102. 908- 14

Radiotckh. Elckıron. (199()) 35(8). 1692-4

J. Vac. Sci. Technol. (199()) B8, 13?3-4

Scanning Tunneling Microscopy Related Methods, NATO ASI Ser., Ser. E (1990) 184, 163-72

Instrum. Exp. Tech. (1990) 33(1), 152-4

J. Appl. Phys. (1990) 67. 7098-110

Prop. II-VI Semicond.:

Bulk Cryst., Epitaxial Films, Quantum Well Struct., Dilutc Magn. Syst., Mater. Res. Soc. Symp. Proc. (1990) 161, 371-6

Ghat k, K. P. Ghoshal, A. Biswas, S. N. Mondal, $\mathrm{M}$

Proc. SPIE-Int. Soc. Opt. Eng. (1990) 1308 (infrared Detect. Focal Plane Arrays), 356-76

Gherm, V. E. Mileshkina, N. V. Semykina, E. A.
J. Phys.: Condens. Matter (1990) 2 , 1263-70 
21 Diffusion of adsorbates on metal surfaces

22 High $\Phi$ values in scanning tunneling microscopy: Ficld emission and tunnel regimes

23 High-resolution simulation of fieldcmission

24 Silicon field emission points for vacuum ICs by wet chemical etching

25 Experimental study of beam emittance and brightness from a high-power field-emission electron gun

26 Study of microfabrication of ultra small ficld emitter with focused ion beam

27 FEM (ficld electron microscopy) observation of single adatoms

28 Some effects in ficld clectron emission from a barium copper yttrium oxide $\left(\mathrm{YBa}_{2} \mathrm{Cu}_{3} \mathrm{O}_{7-\mathrm{x}}\right)$ superconductor

29 Field-emission from a metal covered with a semiconducting layer-a model calculation

30 Long pulse cesium iodide impregnated field emission cathodes
Gomer, R.

Rep. Prog. Phys. (1990) 53. 917-1002

Gomez-Herrero, I.

Gomez-Rodrigue», J. M.

Garcia, R.

Baro, A. M.

Hermannsfeldt, W. B.

Becker, R.

Nucl. Inst. (1990) A298.

Brodic, I.

Rosengreen, A.

Spindt, C. A.

Hunt, C. E.

Trujillo, J.

Hwang, C. S.

Yang, M. S.

Lin, F. C.

Wu, M. W.

Hou, W. S.

Ishikawa, J.

Fukayama, N.

Tsuji, $\mathrm{H}$.

Ishizuka, K.

Kalganov, V. D.

Klimova, T. A.

Mileshkina, N. V.

Kiejna, A.

Niedermann, $P$.

Fischer, $\mathrm{O}$.

Kosai, $\mathrm{H}$.

Fisher, A.
Hyomen Kagaku (1990) 11. 442-8

Surf. Sci. (1990) 231.

$139-41$

Appl. Phys. A. (1990) 50. $331-8$

UCRL-CR-103145: Order No. DE90009533, 6 pp. Avail.: NTIS From: Encrgy Res. Abstr. 1990, 15(12),

Abstr. No. 28555 (1990)

Nucl. Inst. (1990) A288, 324-8

Proc. 13th Symp. on ISIAT

'90, Tokyo (1990),

Takagi, T., cd., Ion

Enginecring Society of Japan, 315-8

Rev. Sci. Instrum. (1990) 61. 1880-2 
31 Field-electron total energy analyzer with retarding potential

32 Simulation and design of field emitter array

33 Carbon on platinum: work function changes caused by atomic carbon and by two-dimensional graphite islands

34 Adsorption of $\mathrm{CO}$ on kinked surface areas of $\mathrm{Pt}$ and $\mathrm{Rh}$ : the relation between work function change upon saturated adsorption and the work function of the corresponding clean adsorbent area

35 Diffusion of $\mathrm{CO}$ on $\mathrm{Ni}(111)$ and $\mathrm{Ni}$ (115)

36 Field emission combination system

37 Field cmission and rf breakdown in copper linac structures

38 Simulation and design of field emitters
Kupryashkin, A. S. Rybakov, Yu. L Sheshin, E. P.

Lee, H. C. Huang, R. S.

Li, X, Q. D. Radojicic, T. Vansclow, $R$.

Li, X. Q. D. Vanselow, $R$.

Lin, T.-S. Lu, H.-J. Gomer, R.

Liu, W. Ren, D. M. Huang, G. M. $\mathrm{Hu}, \mathrm{B}, \mathrm{Y}$. Liu, L. M. Chen, H. N. Yc, S. S. Ma. N.

Loew, G. A. Wang, J. W.

Marcus, R. B. Chin, K. K. Yuan, $Y$. Wang, H. J. Carr, W. N.
Instrum. Exp. Tech. (199()) 3.3(1). 155-7

IEEE Elcc. D (1990) 11. 579.81

Surf. Sci. Lett. (1990) 225. L29-32

Surf. Sci. Lett. (1990) 236. L369-71

Surf. Sci. (1990) 234. $251-61$

J. Electron. Sci. Tech. (Chinese) Suppl. 1 (1990) 24

Part. Accel. (1990) 30), 225-30

IEEE Device (1990) 37. 1545-50 
39 Formation of silicon tips with less than $1 \mathrm{~nm}$ radius

40) Method for study of ficld-cmission statistics over a wide current-density range

41 Chemisorption of NO on a Pt surface II. An FEM study

42 Cold cathode field emission using both aluminum-resin and gold-resin coatings on a tungsten substrate

43 A new perspective on the hot-electron emission from metal-insulator microstructures

44 Thermal field emission observation of single-crystal lanthanum hexaboride

45 Study of field-emitting microstructures using a scanning tunneling microscope

46 Process for making field-emission clectron sources and devices with these sources

47 Photofield emission spectroscopy of the tungsten $\langle 100\rangle$ band structure
Marcus, R. B.

Ravi, T. S.

Gmitter, T.

Chin, K.

Liu, D.

Orvis, W. J.

Ciarlo, D. R.

Hunt, C. E.

Trujillo, J.

Maslov, V. I.

Fursei, G. N.

Kocheryzhenkov, A. V.

Miki, $\mathrm{H}$.

Nagasc, $\mathrm{T}$.

Kioka, $\mathrm{T}$.

Sugai, S.

Kawasaki, K.

Mousa, M. S.

Mousa, M. S.

Nagata, $\mathrm{H}$.

Harada, K.

Shimizu, R.

Niedermann, P.

Renner, C.

Kent, A. D.

Fischer, O.

Pribat, D.

Collet, C.

Radon, $\mathrm{T}$. Jaskolka, S.
Instrum. Exp. Tech. (1990) 33(2), 395-9

Surf. Sci. (1990) 231. 142-8

Surf. Sci. (1990) 231, 149-59

J. Appl. Phys. (1990) $\underline{68}$. 3614-8

Fr. Demande (1990) $20 \mathrm{pp}$.

Appl. Phys. Lett. (1990) 56 . 236-8

Surf. Sci. (1990) 225, 10-20

J. Vac. Sci. Technol. (1990) A8, 594-7

Surf. Sci. (1990) 231, 160-4 
48 Ficld clectron cmission from atomic-size microtips

49 Adsorption of holmium atoms on tungsten crystal faces

50) Emitter for field emission and method of making same; metal carrier wire coated with crystals of transition metal compound oxide

51 Image and surface states in the field-emission microscopy

52. Energy distribution and energy dispersion of virtual image states: $\mathrm{Ni}(111)$ surface

53 Surface diffusion of potassium on (100) and (111) germanium planes

54 Theoretical study of collimated field emission of electrons from a point source

55 Manufacture of thermal field-emission electron source
Saenz, J. J.

Garcia, N.

Vu, T. B.

De Raedt, $\mathrm{H}$.

Shakirova, S. A.

Shevchenko, M. A.

Soredal, S. G.

Steslicka, M.

Steslicka, M.

Kucharczyk, R.

Jurczyszyn, $L$.

Suchorski, J. S.

Tekman, E.

Ciraci, S.

Baratoff, A.

Tsunoda, $\mathrm{K}$.

Hagiwara, $\mathrm{H}$.
Scarnning Tunneling

Microscopy Related

Methods, NATO ASI Ser.,

Ser. E (1990) 184, 409-41

Fiz. Tverd. Tela. (1990)

32(3), 688-93

U. S. Patent No. 4933108 (1990)

Prog. Surf. Sci. (1990) $\underline{35}$, 35-45

Surf. Sci. (1990) 231, 52-8

Surf. Sci. (1990) 231, 130-4

Phys. Rev. B (1990) 42, 9221-4

Jpn. Kokai Tokkyo Koho. (1990) 3 pp. 
1 A stress-induced hydride phase in niobium observed with a ficld-ion microscope

2 Ficld evaporation stimulated by a coherent electromagnetic wave

3 A survey of experinental techniques in surface chemicai physics (editorial)

4 Ficld ion microscopy of defects in regions of displacement cascades in $\mathrm{Cu}_{3} \mathrm{Au}$ alloy

5 Behavior of Ir atoms and clusters on Ir surfaces

6 Displacement distribution and atomic jump direction in diffusion of $\mathrm{Ir}$ atoms on the Ir (001) surface

7 Structure transformation of $\mathrm{Ir}$ clusters on Ir surfaces

8 Energetics in structure transformation of Ir clusters on the Ir-surfaces

9 Diffusion of tungsten on stepped tungsten surfaces

10 Temperature-dependent field ionization of phosphorus atoms in silicon

11 Field ion microscope studies of submonolayer rhodium films on (110) tungsten and molybdenum surfaces

12 Direct observations of surface diffusion
Akaiwa, N.

Wada, $\mathrm{M}$.

J. Less-Common Met. (1990) $\underline{160}, 283-94$

Bakhtizin, R. Z.

Valecv, V. G.

Block, J. H.

Pur. A. Chem. (1990) 62 , 2297-2322

Bunkin, A. Yu. Ivchenko, V. A.

Kuznetsova, L. Yu.

Syutkin, N. N. Tsong, $T$. T.

Chen, C. L.

Tsong, $T$. $T$.
Chen, C. L.

Chen, C. L.

Tsong, T. T.

Chen, C. L.

Tsong, $T$. T.

Choi, D.-S.

Kim. S. K.

Gomer, R.

Dargys, A.

Zurauskienc, N.

Zurauskas, $\mathrm{S}$.

Dhanak, V. R.

Bassett, D. W.

Surf. Sci. (1990) 238, 289-92

Fiz. Met. Metalloved. (1990)

(7), 111-8

Phys. Rev. (1990) B41.

12403-12

Phys. Rev. Letl. (1990) 64 . 3147-50

Appl. Phys. A (1990) 51. 405-10

Jpn. J. Appl. Phys. (1990) 29, L498-501

Surf. Sci. (1990) 234, 262-72

Phys. Status Solidi B, (1990) $162,183-92$

Ehrlich, G.
Scan. Microsc. (1990) 4 . 829-42 
13 Adsorption energetics-1 st principles calculations of adatom interactions and induced local latticc-relaxation

14 Versatile apparatus for etching scanning tunneling microscope tips

15 Simulation of field-ion-microscope images for the aluminum-manganese icosahedral phase

16 Ion-beam ctching as a technique for preparation of high-Tc superconductor microtips for field-ion microscopy
Feibelman, P. J.

Ficring, J. O. Ellis, F. M.

Fowler, H. A. Melmed, A. J. Elswijk, H. B.

Givargizov, E. I. Stepanova, A. N. Volobuev, A. A. Pankrashov, A. I. Yakimov, V. A. Zverkov, V. V. Mashkova, E. S. Molchanov, V. A.

Gong, Y. M.

Guseva, M. I. Dolin, D. E. Ionova, E. S. Suvorov, A. L.

Inglesfield, J. E.

Ivchenko, V. A. Syutkin, N. N. Talantsev, E. F.

Kageshima, $\mathrm{H}$. Tsukada, M.

Kellogg, G. L. Feibelman, P. J.
J. Vac. Sci. Technol. (1990) A8, 2548-51

Rev. Sci. Instrum. (1990) 61. 3911-2

Philos. Mag. B (1990) 61. 811-27

Surf. Sci. (1990) 231, 240-6
Chin. Phys. Lett. (1990) 7 $537-40$

Sov. At. En. R. (1990) 69(1) 609-11

Fiz. Met. Metalloved. (1990) (2), $121-7$

Surf. Sci. (1990) 236 , 385-97

Phys. Rev. Lett. (1990) 64. 3143-6, Erratum (1990) 65. 939 mechanism 
23 Surface physics and chemistry in high electric ficlds

Kreuzer, H. J.

Kreuzer, H. J.

Wang, L. C.

Kreuzer, H. J.

Watanabc, $\mathrm{K}$.

Wang, L. C.

Ksenofontov, V. A.

Mikhailovskii, I. M.

Kupersztych, J.

Mischanchuk, B. G.

Lobanov, V. V.

Pokrovskii, V. A.

Morikawa, $\mathrm{H}$.

Osanai, A.

Sano, T.

Chang, K. S.
Springer Ser. Surf. Sci. (1990) 22 (Chem. Phys. Solid Surf. $\underline{8}$ ), 133-58

J. Chem Phys. (1990) 23 , 6065-9

Surf. Sci. (1990) 232 , 379-92

Phys. Mctals (1990) $\underline{8}$, 554-60

Phys. Scr. (1990) 42 $\underline{42}$ 51-7

Khim. Fiz. (1990) 2(8), 1067-70

Oyo Butsuri (1990) 59(2), 170-4

Shinku (1990) 33(3), 150-3

J. Electron. Sci. Tech. (Chinese) Suppl. 1 (1990) 152

Hu, B. Y.

Liu, W.

Ritchic, B.

Bowden, C. M.

Sung, C. C.

$\mathrm{Li}, \mathrm{Y}, \mathrm{Q}$.
Phys. Rev. A (1990) 41 . 6114-8 
33 Negative ion formation by ficld ionization-studied by field ion microscopy and appearance energy measurements

34 Atomic resolution microscopies in materials

Stiller, K. transmission electron microscopy of heavy ion damage in tungsten

36 An ultrahigh vacuum tip transfer system for the scanning tunneling microscopy/field ion microscopy

37 Field ion microscopy of thin films of yttrium barium copper oxide

$\left(\mathrm{YBa}_{2} \mathrm{Cu}_{3} \mathrm{O}_{7-\mathrm{x}}\right)$

38 Local structural variations near twins in $\mathrm{YBa}_{2} \mathrm{Cu}_{3} \mathrm{O}_{7-\mathrm{x}}$

39 Field desorption from superconducting perovskite surfaces

40 A method of observing fine precipitates in alloys with a field-ion microscope

41 Kinetic theory of field evaporation of metals

Ott, K. H.

Raida, $S$.

Schmitz, R.

Stoll, R.

\section{Sarikaya, M.}

Sugihara, K.

Sakai, A.

Akama, Y.

Shoda, N.

Kato, Y. Hof, P. A.

Wada, M.

Wang, L. C.
Rollgen, F. W.

Tokumoto, $\mathrm{H}$.

Murakami, $\mathrm{H}$.

Talantsev, E. F. Ivchenko, V. A. Ignatenko, Yu. G.

Kreindel, Yu. E.

Ponomarev, A. V. Syutkin, N. N.

Van Bakel, G. P. E. M.

Van Engelen, J. P. M.

Bronsveld, $\mathrm{P} . \mathrm{M}$.

De Hosson, J. T. M.

Vlasov, Y. A.

Golubev, O. L.

Syutkin, N. N.

Talantsev, E. F.

Shrednik, V. N.

ISIJ Int. (1990) 30(5), 341-6

Int. J. Mass Spectrom. Ion Processes (1990) 100, 93-104

Ceram. Trans. (1990) 5 (Adv. Charact. Tech. Ceram.), 247-86

Radiat. Eff. Defect: Solids (1990) 115(1-3), 205-15

Rev. Sci. Instrum. (1990) 61, 81-5

Sverkhprovodimost: Fiz., Khim., Tckh. (1990) 3(8), 1718-25

Phys. Rev. B. (1990) 41, 9502-5

Zh. Tekh. Fiz. (1990)

$\underline{60(10)}, 159-63$

Kreuzer, H. J.
Surf. Sci. (1990) 237. 337-46 
42 Structure, stability, and surface diffusion of clusters: $\operatorname{Ir} r_{x}$ on $\operatorname{Ir}(111)$

43 Field-ion microscopic studies of mass transport in atomic-collision cascades

44 The generation of nickel emitter for Zahran, N. F. field ionization
Wang, S. C.

Ehrlich, G.

Zabolotnyi, V. T.

Suvorov, A. L.

Babacv, V. P.
Surf. Sci. (1990) 239 . 301-32

Fiz. Khim. Obrab. Mater. (1990) (3), 17-9

Indian J. Phys. B (1990) $64 \mathrm{~B}(2), 144-8$ 
1 Ficld ionizable cesium metal-clusters from a foil diffusion source

2 High-field ion sources

3 Liquid metal ion source for cluster ions of metals and alloys: design and characteristics

4 SIMS of microelectronic structures using a liquid metal ion gun

5 Current-voltage characteristics of a gas ficld ion source with a supertip

o. Analysis of the ion emission system with a liquid metal ion source

7 Emission properties of alloy gold-beryllium field ion source

8 Eutectic and emitter materials for liquid metal ion source

9 Anticipated performance of achromatic quadrupole focusing systems when used with liquid metal ion sources

10 Effects of viscosity of capillary wave instabilities of a planar liquid-metal surface in an clectric ficld

11 A lithium liquid metal ion source
Aman, C.

Pettersson, J. B. C.

Holmlid, L.

Bcll, A. E.

Jousten, K.

Swanson, L. W.

Bhaskar, N. D.

Klimcak, C. M.

Frucholz, R. P.

Bishop, H. E.

Greenwood, S. J.

Bœrret, R.

Bohringer, $\mathrm{K}$

Kalbitzer, S.

Chu, X.

Wang, $X$.

Drandarov, $\mathrm{N}$.

Nikolov, B.

Fukuda, H.

Harriott, L. R.

Brown, W. L.

Barr, D. L.

Hc, J.

Miskovsky, N. M.

Cutler, P. H.

Chung, $\mathrm{M}$.

Hesse, E.

Nachring, F. K.
Chem. Phys. (1990: 147. 189-97

Rev. Sci. Instrum. (1990) 61, 363-5

Rev. Sci. Instrum. (1990) 61, 366-8

Surf. Interface Anal. (1990) 16, 70-6

J. Phys. D: Appl. Phys.

(1990) 23, 1271-7

Zhejiang Daxuc Xuebao, Ziran Kexueban, (1990) 24(4), 528-34

Phys. Res. (1990) 13 (EPM '89, Energy Pulse Part. Beam Modif. Mater.), 429-31

Jpn. Kokai Tokkyo Koho. (1990) 7 pp.

J. Vac. Sci. Technol. (199()) A8, 3279-83

J. Appl. Phys. (1990) 68, 1475-82

Phys. Res. (1990) 13 (EPM

'89. Energy Pulse Part. Beam Modif. Mater.), 445-7 
12 Factors affecting ion energy distributions in liquid metal ion sources

13 Droplet and cluster ion emission from gallium and indium liquid metal ion sources

14 A new droplet breakup model for dimer ion formation from a gallium liquid metal ion source

15 Intense metal-ion-beam production using an impregnated-clcctrode-type liquid-metal ion source

16 Aluminum ion source emitting by clectric ficld using impregnated needle-like electrode

17 Focused $\mathrm{Ga}$ ion-beam etching of $\mathrm{Si}$ in chlorine gas

18 Mass and energy analyses of a gold-silicon alloy liquid metal ion source

19 A droplet emission mechanism in liquid metal anodes

2) On the onset voltage of liquid metal Mair, G. L. R. ion sources

21 A design of large curre 1 -ion gun employing liquid metal ion source
Hornsey, R. I.

Hornsey, R. Ishitani, $\mathrm{T}$.

Hornsey, R. Ishitani, $T$.

Ishikawa, J. Tsuji, $\mathrm{H}$.

Aoyama, $\mathrm{Y}$. Takagi, T.

Kikuni, M. Yamada, S.

Komuro, M. Watanabc, $N$.

Hiroshima, $\mathrm{H}$.

Kreissig, U.

Kahn, A.

Ruedenauer, F. G.

Steiger, W.

Mair, G. L. R.

Mori, Y.

Wang, $H$.

Endo, $\mathrm{K}$.

Yamauchi, $\mathrm{K}$.

Id́c, $T$.
252 pp. Avail. Univ. Microfilms Int., Order No. BRD-90344 From: Diss. Abstr. Int. B (1990) 51(6), 2945

Jpn, J. Appl. Phys. Part 1 (1990) 29(10), 2116-21

Jpn. J. Appl. Phys. Part 2 (1990) 29(6), L1007-10

Rev. Sci. Instrum. (1990) 61, 592-4

Jpn. Kokai Tokkyo Koho. (1990) 4 pp.

Jpn. J. Appl. Phys. (1990) 29, 2288-91

J. Phys. D: Appl. Phys. (1990) 23, 959-63

J. Phys. D: Appl. Phys. (1990) $23,1239-43$

Nucl. Instrum. Methods Phys. Res. (1989) B43. 240-2, Erratum (1990) B47, 106

Rev. Sci. Instrum. (1990) 61, 1874-9 
22 Design of a large-current liquid metal ion source
Mori, Y.

Wang, $\mathrm{H}$.

Endo, $\mathrm{K}$.

Yamauchi, K.

Ide, $\mathrm{T}$.

Goto, $\mathrm{H}$.

Mori, Y.

Wang, $\mathrm{H}$.

Endo, K.

Yamauchi, $\mathrm{K}$.

Ide, $T$.

Goto, $\mathrm{H}$.

Pregenzer, A. L.

Pregenzer, A. L.

Bieg, K. W.

Olson, R. E.

Panitz, J. A.

Rao, S.

Bell, A. E.

Schwind, G. A.

Swanson, L. W.

Rao, S.

Bell, A. E.

Schwind, G. A

Swanson, L. W.

Read, P. M.

Maskrey, J. T.

Alton, G. D.

Schwieters, J.

Zehnpfenning, J. F.

Nichuis, E.

Benninghoven, A.
Technol. Rep. Osaka Univ. (1990) 40(1986-2003), 103-11
Rev. Sci. Instrum. (1990) 61. 3412-5
Rev. Sci. Instrum. (1990) 61, 571-3

J. Appl. Phys. (1990) 67. 7556-9

J. Vac. Sci. Technol. (1990) A8, 2258-64

J. Vac. Sci. Technol. (1990) B8, 1932-6

Rev. Sci. Instrum. (1990) 61. 502-4

J. Vac. Sci. Technol. (1990) A8, 3752-7 of a liquid metal field ion
short HV-extraction pulses

Szep, J.

Szilagyi, M.

J. Vac. Sci. Technol. (1990) A8, 3758-62 
31 Theoretical study of the ion energy distribution in Ga LMIS by the Boltzmann equation II. Effect of the ion density distribution

32 Extraction of $\mathrm{mA}$ class metal ion beams from multipoint emission of impregnated-electrode-type liquid metal ion source

33 Stochastic effects occurring after ion cmission from liquid metal ion sources

34 Beam dynamics of a liquid metal ion source

35 Liquid-metal ion source

36 Structure of liquid-metal ion source

37 Design of a high-resolution focused ion beam system using liquid metal ion source
Tanitsu, $\mathrm{H}$.

Kosuge, $\mathrm{T}$.

Makabe, T.

Tsuji, $\mathbf{H}$.

Aoyama, Y.

Ishikawa, J.

Ward, J. W.

Kubena, R. L.

Whealton, J. H. Meszaros, P. S.

Rothe, K. E.

Raridon, R. J.

Ryan, P. M.

Yamada, S. Amamiya, $M$. Hikita, Y.

Yamada, S. Amamiya, $M$. Hikita, Y.

Zhou, L.

Orloff, J.
Proc. 13th Symp. on ISIAT

'90, Tokyo (1990),

Takagi, T., ed., Ion

Engincering Socicty of

Japan, 67-70

Proc. 13th Symp. on ISIAT

'90, Tokyo (1990),

Takagi, T., ed., Ion

Engincering Society of

Japan, 59-66

J. Vac. Sci. Technol. (1990) B8, 1923-6

Rev. Sci. Instrum. (1990) 61. 568-70

Jpn. Kokai Tokkyo Koho. (1990) 4 pp

Jpn. Kokai Tokkyo Koho. (1990) 4 pp.

J. Vac. Sci. Technol. (1990) B8, 1721-4 
Addendum to Atom Probe Field Ion Microscopy and Related Topics: A Bibliography for 1978-1987, 1988, and 1989

$1 \quad$ Ficld emitted electron inclastic energy losses spectroscopy

2 Adsorbate mobility investigation by means of ficld emission fluctuation method

3 Ficld emission work function of the $\mathrm{Ni} / \mathrm{W}(110)$ systcm

Size effects in metallic clusters using field emission and field ion microscopy

5 Model of the epitaxy of metals on metals based on the field electron emission microscopy studies

A new method to calculate the emission current of liquid metal ion sources

7 Microanalysis by ion microanalyzer with liquid metal ion source

8 Pulsed laser atom probe analysis of semiconductor materials
Bakhtizin, R. Z.

Valeev, V. G.

Kukharenko, Yu. A.

Beben, J.

Meclewski, R.

Blaszczyszyn, R.

Blaszczyszynowa, $M$.

Castro, T. K.

Ciszewski, A.

Cui, Z

Tong, $\mathrm{L}$.

Gennai, N.

Nakazawa, S.

Ikeda, T.

Grovenor, C. R. M.

Cerezo, A.

Liddle, J. A.

Smith, G. D. W.
Izv. Khim. (1989) 22(3-4), 392-6

Proc. 3rd Conf. Surface Physics (1989) $\mathrm{V}, 48-51$, Kapelewski, J., Rutkowski, J., Solarz, L., eds.,

Wydawnictiwo Uniwersytetu

Lodzkicgo, Lodz, Poland

Proc. 3rd Conf. Surface Physics (1989) V, 56-9, Kapelewski, J., Rutkowski, J., Solarz, L., eds., Wydawnictwo Uniwersytetu Lodzkicgo, Lodz, Poland

(1989) 212 pp., Avail. Univ. Microfilms Int., Order No. DA9018797

Proc. 3rd Conf. Surface Physics (1989) $\underline{V}, 102-5$, Kapelewski, J., Rutkowski, J., Solarz, L., cds.,

Wydawnictwo Uniwersytelu Lodzkicgo, Lodz, Poland

Zhenkong Kexue Yu Jishu (1989) 9(6), 364-8

KOBELCO Technol. Rev. (1989) 6, 18-20

Inst. Phys. C (1987) 87 , 665-74 
9 Recent developments in atom probe nuicroanalysis of materials with ultrafine microstructures

10 Nucleation, growth, and coarsening of $\gamma^{\prime}$-precipitates in Ni-5.0 at. \% Al-5.8 at. \% $\mathrm{Ti}$

11 An atomistic study of the GaAs-Pd interface

12 Composition fluctuations in compound semiconductors

13 Formation and decomposition of ionic associates of methyl alcohol during field ionization

14 Active-gas discharge cleaning and low-field electron emission

15 Construction of combined field ion-scanning tunneling microscope and its performances

16 Fluorine gas ficld ion source
Grovenor, C. R. M.

Cerezo, A.

Sassen, J.

Liddle, J. A.

Hetherington, M. G.

Mackenzic, R. A. D.

Shollock, B. A.

Smith, G. D. W.

Hein, W.

Kobayashi, A.

Sakurai, T.

Hashizume, T.

Sakata, T.

Liddle, J. A.

Mackenzic, R. A. D.

Grovenor, C. R. M.

Lobanov, V. V.

Golovatyi, V. G.

Korol, E. N.

Malev, M. D.

Weisser, D. C.

Murakami, Kenji

Miyao, Masahiro

Nomura, Takashi

Ishikawa, Kenji

Hagino, Minoru

Sasaki, Akira

Yamaguchi, Tsuyoshi

Nishino, Naoya

Fukuoka, Shiuchi

Ochiai, Y

Nomura, E.

Kojima, Y.

Matsui, S.
Inst. Phys. Conf. Ser.

No. 98: Chapter 4 (1989)

147-52

Acta Metall. (1989) $\underline{37}$.

2145-52

J. Appl. Phys. (1986) 59.

3448-53

Microscopy of

Semiconducting Materials

VI (1989) 81-6, Cullis, A. G. and Hutchison, J. C., eds.,

Inst. Phys., Bristol, UK

Teor. Eksp. Khim. (1989)

25, 653-9

IEEE Trans. Elect. Insul. (1989) 24, 1019-21

Shizuoka Daigaku Denshi Kogaku Kenkyusho Kenkyu Hokoku (1989) 24(2), 79-90

Proc. 1989 Intern. Symp. on MicroProcess Conf., Jpn. J. Appl. Phys. (1989), 298-302 
Addendum to Atom Probe Field Ion Microscopy and Related Topics: A Bibliography for 1978-1987, 1988, and 1989

17 Electron scattering assisted by an intense electromagnetic field: Exact solution of a simplified model

18 Microclectronic field ionizer and its fabrication

19 Ficld-ion microscopy of crystals of $\mathrm{RBa}_{2} \mathrm{Cu}_{3} \mathrm{O}_{7-x}(\mathrm{R}=\mathrm{Y}, \mathrm{Tb}, \mathrm{Dy}, \mathrm{Eu})$

20) Liquid metal ion sources

21 Comment on "Field penetration and band bending near semiconductor surfaces in high clectric ficlds" by T. T. Tsong
Sacks, R. A.

Szoke, A.

Spindt, C. A.

Talantsev, E. F. Ivchenko, V. A.

Syutkin, N. N.

Samokhvalov, A. A.

Chebotaev, N. M.

'Thompson, S. P.

Tomita, M

Kuroda, T.
Phys. Rev. (1989) A40. 5614-32

Eur. Pat. Appl. (1989) $12 \mathrm{pp}$.

Sverkhprovodimost: Fiz., Khim., Tekh. (1989) 2(12), 123-30

(1982) 158 pp., Avail. Univ. Microfilms Int., Order

No. BRD-89458

Surf. Sci. Letl. (1989) 222 . L801-3 


\section{APPENDIX}

The reports and dissertations listed in the bibliography may generally be obtained through one of the following agencies:

National Technical Information U. S. Department of Commerce 5285 Port Royal Road

Springfield, VA 21161 USA

Telephone: (703) 487-4650

University Microfilms Int.

Dissertation Information Service

300 North Zeeb Road

Ann Arbor, MI 48106 USA

Telephone: (800) 521-3042 (Toll Free)

or

(313) $761-4700$ 


\section{INTERNAL DISTRIBUTION}

\section{1-2. Central Research Library}

3. Document Reference Section

4-5. Laboratory Records Department

6. Laboratory Records, ORNL RC

7. ORNL Patent Section

8-10. Metals and Ceramics Records Office

11. Alexander, K. B.

12. Alexander, M. B.

13. Bentley, J.

14. Bloom, E. E.

15. Horton, J. A.

16. Horton, L. L.

17. Hulett, L. D.

18. Jayaram, Raman

19. Kenik, E. A.

20. Mantovani, J. G.

21-70. Miller, M. K.

71. More, K. L.

72-74. Russell, K. F.

75. Stiegler, J. O.

\section{EXTERNAL DISTRIBUTION}

76. Prof, Jochen H. Bloch

Fritz-Haber-Institut MPG

Faradayweg 4-6

D-W-1000 Berlin 33

Germany 
77. Dr. Piotr S. Bragiel Institute of Physics

Pedagogicol University of Czestochowa

Armii Krajowej 13/15

42-201 Czestochowa

Poland

78. Dr. Patrick P. Camus

Applied Superconductivity Center

University of Wisconsin

1500 Johnson Drive

Madison, WI 53706

USA

79. Dr. Alfred Cerezo

Department of Materials

University of Oxford

Parks Road

Oxford OXI 3PH

United Kingdom

80. Dr. Wolfgang Drachsel

Fritz-Haber-Institut MPG

Faradayweg 4-6

D-W-1000 Berlin 33

Germany

81. Prof. Luo Enze

Department of Physics

Xidian University

Xi'an 710071

China

82. Dr. Norbert Ernst

Fritz-Haber-Institut MPG

Faradayweg 4-6

D-W-1000 Berlin 33

Germany

83. Mr. Larry A. Fletcher

4663 Solomons Island Road

Harwood, MD 20776

USA

84. Dr. Richard G. Forbes

Department of Electronic and Electrical Engineering

University of Surrey

Guildford, Surrey GU2 5XH

United Kingdom 
85. Dr. Kazuhiro Hono

Institute for Materials Rescarch

Tohoku University

2-1-1 Katahira, Aoba-ku

Sendai 980

Japan

86. Mr. Jonathan Hyde

Department of Materials

University of Oxford

Parks Road

Oxford OXI 3PH

United Kingdom

87. Dr. Yuichi I. Ishikawa

Mechanical Engineering Rescarch Laboratory, Hitachi Ltd.

502 Kandatsu

Tsuchiura-shi

Ibaraki 300)

Japan

88. Dr. Gary L. Kcllogg

Sandia National Laboratories

Division 1114

P. O. Box 5800)

Albuquerque, NM 87185

USA

89. Prof. Erich A. P. Krautz

Institut für Festkörperphysik

T. U. Graz.

Petersgasse 16 A-8010

Graz

Austria

90. Dr. Anatoli N. Kudriavtsev

I. P. Bardin Central Research Institute,

Iron and Stcel Industry

9/23 2nd Baumanskaja

Moscow 107005

USSR

91. Dr. Manfred Leisch

Institut für Festkörperphysik

T. U. Graz

Petersgasse 16 A-8010

Graz

Austria 
92. Prof. Evgeny A. Litvinov Institute of Electrophysics USSR Academy of Sciences 34, Komsomolskaya Str. Sverdlovsk 620219 USSR

93. Dr. Guangyi Y, Liu Electronics Research Institute Chinese Academy of Sciences 17 Zhong-Guan-Cur Road Beijing 100080 China

94. Dr. R. A. D. Mackenzic Department of Materials University of Oxford Parks Road Oxford OXI 3PH United Kingdom

95. Dr. Alain Menand Laboratoric de Microscopie Ionique UA CNRS 808 University de Rouen Faculte Des Sciences BP 118 Mt. St. Aignan 76134 France

96. Dr. Hiroshi Morikawa Nagoya Institute of Technology Gokosco-cho, Showa-ku Nagoya 466 Japan

97. Dr. Marwan S. Mousa Department of Physics Mu'tah University P O Box 7 Mu'tah Ai-karak Jordan

98. Prof. Klaus Müller

Lchrstuhl f. Festkörperphysik Universität Erlangen-Nürnberg Staudtstr 7 D-8520

Erlangen

Germany

99. Prof. Osamu Nishikawa

The Graduate School at Nagatsuta

Tokyo Institute of Technology 4259 Nagatsuta Midori-ku, Yokohama 227

Japan 
100. Dr. Elichi Nomura Aono Atomcraft Project Kaga 1-7-13, Itabashi-ku Tokyo 173

Japan

101. Dr. Hans F. Norden Department of Physics Chalmers University of Technology Göteborg S-41296 Sweden

102. Dr. V. E. Ptitsin Institute for Analytical Instrumentation USSR Academy of Sciences 26 Ogorodnicova Leningrad 198103 USSR

103. Dr. Ulf A. S. Rolander Department of Physics Chalmers University of Technology Götcborg S-41296 Sweden

104. Dr. Sergei I. Shkuratov Institute of Electrophysics USSR Academy of Sciences 34, Komsomolskaya Str. Sverdlovsk, 620219 USSR

105. Dr. George D. W. Smith Department of Materials University of Oxford Parks Road

Oxford OXI 3PH

United Kingdom

106. Mr. Jörg Unger

Fritz-Haber-Institut MPG

Faradayweg 4-6

D-W-1()0) Berlin 33

Germany

107. Dr. Nelia Wanderka

Hahn-Meitner -Institut, N5

Glienicker Str. 100)

W-1000 Berlin 39

Germany 
108. Dr. Robert Waugh

VSW Technology Lid.

Albert Drive, Burgess Hill

W. Sussex RH15 9NX

United Kingdom

109. Prof. Masahiko Yamamoto

Department of Materials Science and Engineering

Osaka University

2-1 Yamadaoka Suita

Osaka 565

Japan

110. DOE Field Office,

Oak Ridge, Assistant Manager for

Energy Researsh and Development

PO Box 2001

Oak Ridge TN 3783 ! USA

111-120. Department of Energy

Office of Scientific and

Technical Information

Office of Information Service

PO Box 62

Oák Ridge TN 37831 USA

For distribution by microfiche as shown in DOE/TIC-4500

Distribution Category UC-404 

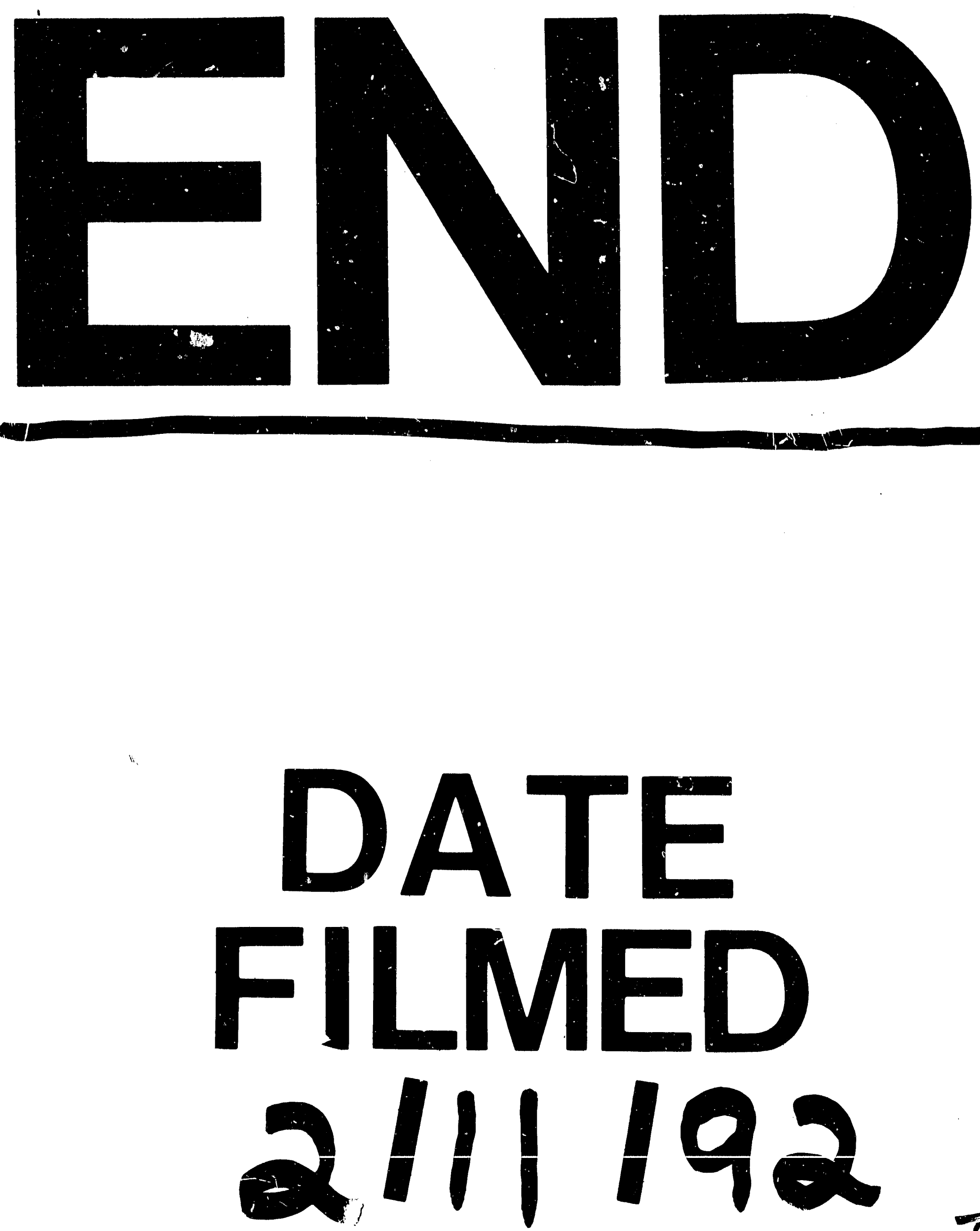
